\title{
METABOLIC PATHWAY MEMBERSHIP INFERENCE USING AN ONTOLOGY-BASED SIMILARITY APPROACH
}

\author{
by \\ Imam Cartealy
}

A thesis submitted to the Faculty of the University of Delaware in partial fulfillment of the requirements for the degree of Master of Science in Bioinformatics and Computational Biology

Fall 2019

(C) 2019 Imam Cartealy

All Rights Reserved 


\title{
METABOLIC PATHWAY MEMBERSHIP INFERENCE USING AN ONTOLOGY-BASED SIMILARITY APPROACH
}

\author{
by \\ Imam Cartealy
}

Approved:

Li Liao, Ph.D.

Professor in charge of thesis on behalf of the Advisory Committee

Approved:

Cathy Wu, Ph.D.

Director of Center for Bioinformatics and Computational Biology

Approved:

Levi Thompson, Ph.D.

Dean of the College of Engineering

Approved:

Douglas J. Doren, Ph.D.

Interim Vice Provost for Graduate and Professional Education and Dean of the Graduate College 


\section{ACKNOWLEDGMENTS}

I would like to express my sincere thanks to my advisor, Prof Li Liao and Prof Cathy $\mathrm{Wu}$ who have been giving guidance and encouragement during the thesis project. This thesis could not have been possible without their tremendous effort and support. I grateful to the University of Delaware and Fulbright to support me during the study. I also truly appreciate to my wife and family whose love, support and understanding kept me going. 


\section{TABLE OF CONTENTS}

LIST OF TABLES . . . . . . . . . . . . . . . . . . . . . v v

LIST OF FIGURES . . . . . . . . . . . . . . . . . . . . . . . v vi

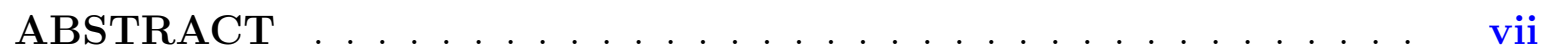

\section{Chapter}

1 INTRODUCTION . . . . . . . . . . . . . . . . 1

2 RELATED WORKS . . . . . . . . . . . . . . . . . 6

3 DATA AND METHOD . . . . . . . . . . . . . . . 12

3.1 The GO dataset and gene annotation . . . . . . . . . . . . . . . 12

3.2 UniProt dataset . . . . . . . . . . . . . . . . . . . 12

3.3 The KEGG pathway database . . . . . . . . . . . . . . . . . . . 12

3.4 Gene representation . . . . . . . . . . . . . . . . . . . . . . . . . . . . . . . . . . .

3.5 Pathway membership prediction . . . . . . . . . . . . . . . 15

3.6 Performance evaluation . . . . . . . . . . . . . . . . . . 15

4 RESULTS AND DISCUSSIONS . . . . . . . . . . . . . 17

4.1 Random gene pair semantic similarity distribution . . . . . . . . . . 17

4.2 Metabolic pathway membership prediction . . . . . . . . . . . . . 18

4.3 Distribution of high semantic similarity score . . . . . . . . . . . . 28

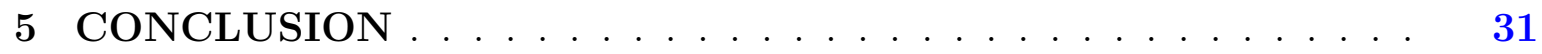

REFERENCES . . . . . . . . . . . . . . . . . . . 33

Appendix

KEGG METABOLIC PATHWAY . . . . . . . . . . . . . . 36 


\section{LIST OF TABLES}

2.1 Similarity measures and their mixing strategy found in literature . 9

4.1 The average similarity score of 10000 random pair genes . . . . . 18

4.2 The area under the curve ROC of semantic similarity measure for BP, MF and CC ontology . . . . . . . . . . . . . . . . . . 19

4.3 Improvement of our measure against other similarity measures in BP, $\mathrm{CC}$ and MF ontology (in percentage) . . . . . . . . . . 22

4.4 Performance of measures in predicting genes membership of metabolic pathways that contains different number of genes . . . . 24

4.5 The ratio of semantic similarity scores . . . . . . . . . . . 25

4.6 ROC score for combine ontologies . . . . . . . . . . . . . . 26

4.7 Running time of similarity measures in seconds . . . . . . . . . 27

4.8 Total number of pairs in regard of their gene's member status in the pathway ............................ 29

A.1 List of KEGG Metabolic Pathway . . . . . . . . . . . . . 36 


\section{LIST OF FIGURES}

1.1 Schematic graph of metabolic pathway . . . . . . . . . . 2

4.1 ROC curve plot for gene membership prediction . . . . . . . . . 20

4.2 Distribution of gene family, interacting, and synonym genes in the

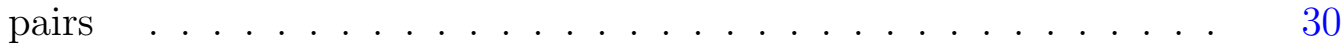




\begin{abstract}
Determining whether a protein belongs to a metabolic pathways is an important annotational task, can provide context to the basic functional annotation and aid reconstruction of incomplete pathways. In this work, we develop a method for pathway membership inference based gene ontology (GO) similarity between a query protein and proteins that are known to the members of a a given pathway. We specifically use human metabolic pathway from KEGG and human gene annotation dataset from Gene Ontology in this experiment. By comparing with various existing GO term semantic similarity, we develop an effective and efficient way to take into both information content of individual GO terms and the whole GO hierarchy. We test the classifier using 10-fold cross validation for all metabolic pathways reported in KEGG database and demonstrate that our method either outperform with statistical significance or perform comparably with a suite of existing semantic similarity measures, as evaluated using ROC score. And our method outperforms other methods in running time by multiple orders of magnitude for long pathways.
\end{abstract}




\section{Chapter 1 INTRODUCTION}

A metabolic pathway is a chain of biochemical reactions in the cell. It consists of chemical compounds and protein enzymes where protein enzymes catalyzes the conversion from substrate compounds into product compounds. The reaction in metabolic pathway then can be represented as a graph where each protein enzyme and its corresponding substrate and product compound are connected by edges (figure 1.1(a)). The enzymatic reaction is not isolated, but interrelated where the product of reaction $\mathrm{A}$ can be the substrate of reaction $\mathrm{B}$ and it can be represented as a graph of compound/substrate network (figure 1.1(b)). From protein perspective, a metabolic pathway can be viewed as a list of proteins in the pathway. These proteins can be represented as a graph where the protein enzymes are the vertices. Two proteins are connected by the edge if the product compound of first protein enzyme is the substrate compound of the second protein enzyme (figure 1.1(c)). As a result, we can model metabolic pathway from the proteins (or genes) as a graph by using this approach. 
Figure 1.1: Schematic graph of metabolic pathway

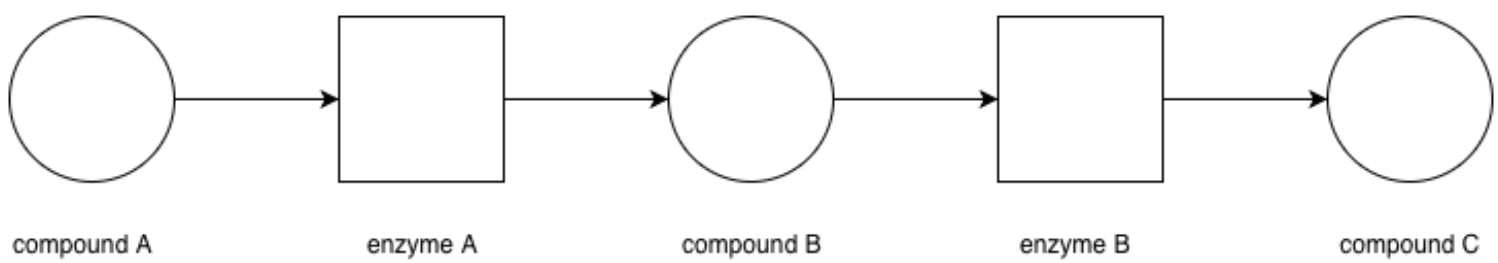

(a)

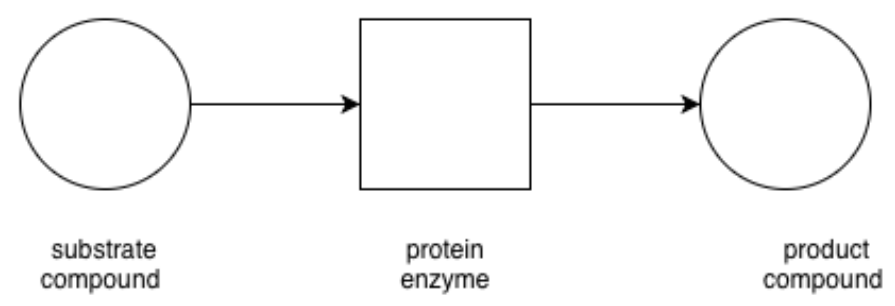

(b)

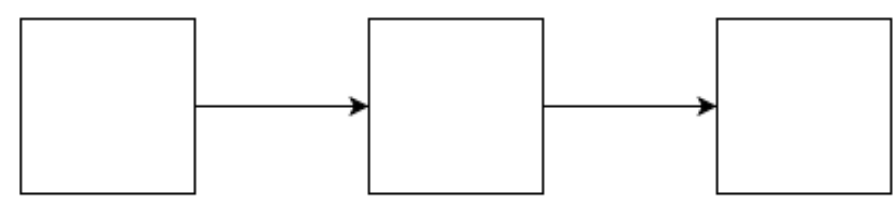

enzyme A

enzyme B

enzyme B

(c)

Metabolic pathway network can be viewed as (a) the whole graph of chemical compound and protein enzyme, (b) enzymatic reaction which connect substrate and the product, or (c) a graph of protein (enzyme) 
Identification of metabolic pathway is one of important task in biological science since understanding the metabolic profiles is needed to uncover the underlying cellular physiology. Any subtle changes in metabolic pathway may lead to the development of abnormal cell or disease. For example, changes in expression of metabolites can lead to colorectal cancer (Brown et al. (2016)) and lung cancer (Cameron et al. (2016)) . Certain diseases such as phenylketonuria and Lesch-Nyhan syndrome are caused by the lack of enzymes that cause the disruption of metabolic pathways. The loss of phenylalanine hydroxylase in phenylketonuria patients can cause mental retardation, organ damage, unusual posture.

The Identification of metabolic mechanisms in laboratory involves many laborious experimental techniques mainly in characterization of protein enzymes in metabolic pathways. It requires advance technology, expensive equipment, and highly skills manpower to perform the experiments. Another approach is to use computational methods for modeling the pathway and to infer specific task by using the model. Pathway membership inference is to determine whether a protein is a member in the list of a given pathway. This is an important annotation task that can not only provide context to the basic function annotation of proteins but also more importantly aid reconstruction of incomplete metabolic pathways, which can subsequently help better understand metabolism and physiology of cells and provide complementary perspective to study evolutionary.

However, traditional sequence similarity based homology approaches to characterizing proteins for their enzymetic properties run into difficulties when sequence identity is lower than 60\% (Radivojac et al. (2013)) due to multiple hits of homologous proteins. Facing this challenge, various efforts have been made to go beyond individual proteins and their homologs to leverage the large amount of annotations for proteins in their functional context.

This context may come from two perspectives. One perspective is based on gene ontology (GO) (Duan et al. (2006)), which provides a hierarchy of controlled terms define protein functions with varied levels of specificity for different cellular 
functions/processes. The other perspective comes from metabolic pathways, or proteins that serve as enzymes catalyzing the reactions in a given pathway. While these proteins may not have the same function (thus with different GO terms), they function in a coordinated way to enable the pathway and hence possess certain functional relatedness (Chitale et al. (2011)). To expand on this concept, the counted co-occurrences of GO terms are used to score the functional relatedness or coherence and further to assign proteins to pathways (Chitale et al. (2011)).

For the GO term perspective, various similarity measures have been developed to quantify the semantic similarity of GO terms and applied it in quantitative comparison of functional similarity of gene products, although most of these methods are not developed for metabolic pathway membership inference. Essentially, those measures mainly involve two steps: 1) calculation of the term similarity, and 2) calculation of the gene product similarity. In the first step, the semantic similarity between two GO term is calculated to incorporate the GO hierarchy, via the following three approaches: edge-based, node-based, or a hybrid of the former two.

The edge-based approach relies on the structure of the GO graph (Wu et al. (2006), Chen et al. (2007), Al-Mubaid \& Nagar (2008)). Basically, the semantic similarity between two GO terms is defined as the distance between two connected terms, which is the length of a path connecting the two terms in the GO graph. Since there may exist more than one path connect the two terms, this approach usually use the most recent common ancestor (MRCA), or the closest common ancestor to both terms, to quantify the semantic similarity between terms. The simplest method of this approach is by using directly the path length between two terms and convert it to similarity (Al-Mubaid \& Nagar (2008)). However, this simple method suffers from shallow annotation problem (Guzzi et al. (2012)), in which the similarity of two term near the GO root term is very high. Another problem arises from the assumption that GO terms at the same level in the graph have the same specificity, which in biological ontology is not always true since different gene properties have different detailed information (Song et al. (2014)). Therefore, the same level of terms in the GO graph may have 
different weight. Thus, more advanced techniques of edge-based approach such as RSS considers global structure of the DAG graph by assigning different weights of edges at different level.

The node-based approach focuses on information that is contained in the node. Methods in this approach such as Resnik's (Resnik (1999)), Lin's measure (Lin (1998)) , and Jiang's and Conrath's measure (Jiang \& Conrath (1997)) rely on information content (IC). IC is defined as the negative log of term frequency in a given annotation corpus, such as Gene Ontology Annotation (GOA). Given two GO terms, the information contained in the common ancestors is used alone or in combination with other terms to quantify the similarity between the two terms. Since it is likely that there are multiple common ancestors, most methods use the maximum informative common ancestor (MICA) which is the common ancestor that contain the maximum IC. Other methods, such as simGIC (Pesquita et al. (2007)) considers not only MICA in determining the gene similarity, but also all ancestor contributions to the similarity measurement of two terms. Unfortunately, similar to the edge-based approach, node-based approach also suffers from shallow annotation problem, especially when the MICA is located near the root term.

The third approach combine the node- and edge-based approaches. Essentially, this hybrid approach considers both the information contained in the nodes and the structure of the GO graph using path distance as weight (Chen et al. (2007), Wu et al. (2013), Ehsani \& Drabløs (2016)), in order to minimize the problem found in edgeand nod-based approach alone.

In this work, we proposed a method for metabolic pathway membership inference, bringing together both perspectives. From the pathway perspective, proteins from an incomplete pathway provide a "model" that a query protein needs to fit in, and the similarity of GO terms for individual proteins provide some measure for functional relatedness. With respect to GO term similarity, our approach is a hybrid one, taking into account of both nodes information and GO graph topology, with a consideration of computational efficiency. 


\section{Chapter 2}

\section{RELATED WORKS}

There are many methods that have been developed for calculating GO semantic similarity. One of the first method is Lord (Lord et al. (2003)) which applied Resnik similarity in GO term. The Resnik similarity itself relies on the information content(IC) that is contained by the terms. The IC is defined as negative log of term frequency in given corpus as:

$$
I C(t)=-\log [p(t)]
$$

where $p(t)$ is term frequency of $t$ in a given annotation corpus, such as Gene Ontology Annotation (GOA). To find similarity between two terms, Resnik similarity measure finds the maximum IC of common ancestor, as:

$$
\operatorname{sim}\left(t_{i}, t_{j}\right)=\max _{t \in P\left(t_{i}, t_{j}\right)} I C(t)
$$

where $t$ is the common ancestor of $t_{i}$ and $t_{j}$.

Wang is an edge-based approach that relies on the structure of GO term. Wang similarity measure (Chen et al. (2007)) considers contributions from all ancestor terms to a given term in determining the semantic value of term by using the distance between the two terms. Terms closer to term $t_{i}$ contribute more to semantic value of term $t_{i}$. Contribution of term $t_{i}$ to itself is 1 , while contribution of terms $t_{p_{i}}$ to semantic value 
of term $t_{i}$ is determined by semantic contribution of all $t_{p_{i}}$ children term t' weight by semantic contribution factor $w_{e}$. Formally Wang semantic value $\mathrm{S}$ is defined as:

$$
\left\{\begin{array}{l}
S_{t_{i}}\left(t_{i}\right)=1 \\
S_{t_{i}}\left(t_{p_{i}}\right)=\max \left\{w_{e} * S_{t^{\prime}}\right\}\left|t^{\prime} \in \operatorname{childrenof}\left(t_{p_{i}}\right)\right| i f t_{p_{i}} \neq t_{i}
\end{array}\right.
$$

where $t_{p_{i}}$ is ancestor term of $t_{i}, w_{e}$ is weight of edges, $t^{\prime}$ is children of $t_{p}$. Then the semantic contribution of all ancestor terms of $t_{p_{i}}$ to $t_{i}$ is defined as:

$$
S V\left(t_{i}\right)=\sum_{t \in T_{p_{i}}} S_{t_{i}}\left(t_{p_{i}}\right)
$$

where $T_{p_{i}}$ is all ancestor term of $t_{i}$. The semantic similarity between term $_{i}$ and term $m_{j}$ then is defined as:

$$
S\left(t_{i}, t_{j}\right)=\frac{\sum_{t \in T_{p_{i}} \cap T_{p_{j}}}\left(S_{t_{i}}(t)+S_{t_{j}}(t)\right)}{S V\left(t_{i}\right)+S V\left(t_{j}\right)}
$$

RSS is also an edge-based similarity measure. Unlike other edge-based similarity measures, RSS considers both of the graph structure above (generality) and below (specificity) a given term. To quantify the similarity measure, RSS defines three definitions: $\alpha$ which defines the specificity, $\beta$ defines generality, and $\gamma$ defines local distance between two terms and the MRCA . The specificity is defined by the number of common parent terms between the two terms, and formally defined as:

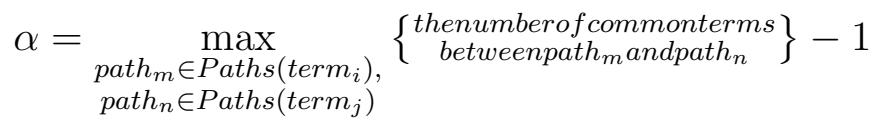

where Paths $\left(\right.$ term $\left._{i}\right)$ and Paths $\left(\right.$ term $\left._{j}\right)$ are the paths in the graph that are induced from term $_{i}$ and term $_{j}$ respectively. The generality $\beta$ is defined as the minimum distance between the term, and its leaf terms (term without any descendant):

$$
\beta=\max \left\{\min _{u i n U}\left\{\operatorname{dist} \text { term }_{i}, u\right)\right\}, \min _{v \in V}\left\{\operatorname{dist}_{\left.\left(\text {term }_{j}, v\right)\right\}}\right\}
$$


where $U$ and $v$ are all leaf nodes descending from term $t_{i}$ and term $t_{j}$ respectively. $\gamma$ is defined as:

$$
\gamma=\operatorname{dist}\left(M R C A, \operatorname{term}_{i}\right)+\operatorname{dist}\left(M R C A, \text { term }_{j}\right)
$$

where MRCA is the most recent common ancestor of term $t_{i}$ and $t_{j}$

Then similarity measure between two terms is define as:

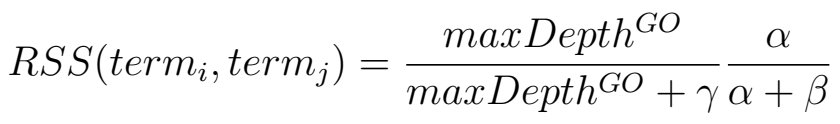

where maxDepth ${ }^{G O}$ is maximum distance between the root term and leaf term.

TopoICSim (Ehsani \& Drabløs (2016)) is a hybrid-based similiraty measure which uses both IC and topology of GO terms in measuring term similarity. First it calculates the weighted distance between two terms. Given two terms, there are multiple path that connect two terms. TopoICSim uses both the shortest path and longest path as define by:

$$
\begin{aligned}
& w S P\left(t_{i}, t_{j}\right)=\underset{P \in A\left(t_{i}, t_{j}\right)}{\operatorname{argmin}} \operatorname{IIC}(P) \operatorname{xlen}(P) \\
& w L P\left(t_{i}, t_{j}\right)=\underset{P \in A\left(t_{i}, t_{j}\right)}{\operatorname{argmax}} \operatorname{IIC}(P) \operatorname{xlen}(P)
\end{aligned}
$$

where $I I C$ is inverse $\mathrm{IC}$, and $P$ is all paths that connect $t_{i}$ and $t_{j}$. To determine the distance between the two terms, TopoICSim uses a subset of common ancestors called disjunctive common ancestor as defined by:

$$
\operatorname{DiscComAnc}\left(t_{i}, t_{j}\right)=\left\{x \in \operatorname{ComAnc}\left(t_{i}, t_{j}\right) \mid P(x, \text { root }) \cap C(x)=\emptyset\right\}
$$

where $\operatorname{ComAnc}\left(t_{i}, t_{j}\right)$ is common ancestors between $t_{i}, t_{j}, P(x$, root $)$ is the path between ancestor term $x$ and root, and $C(x)$ is set of all immediate children of $x$. The distance of the two term through disjunctive common ancestor is defined as the ratio between the shortest weighted distance of two terms through the disjunctive common 
ancestor $x$ and the weighted longest distance between $x$ and the root as defined by:

$$
D\left(t_{i}, t_{j}\right)=\min _{x \in \operatorname{DisComAnc}\left(t_{i}, t_{j}\right)} \frac{w S P\left(t_{i}, x\right)+w S P\left(t_{j}, x\right)}{w L P(x, \text { root })}
$$

The distance of two terms is then transformed into similarity by:

$$
S\left(t_{i}, t_{j}\right)=1-\frac{\operatorname{Arctan}\left(D\left(t_{i}, t_{j}\right)\right)}{\pi / 2}
$$

There are other semantic similarity measures that do not use either the information content or the structure of the graph. CAS (Chitale et al. (2011)) is based on counting the term frequency, which is usually used in natural language problems. Specifically, CAS quantifies the GO term semantic similarity using PMI, the likelihood of finding two terms together in given corpus. Similarly, CAS calculates the probability of given two term presents together in given corpus as semantic similarity between two terms as :

$$
C A S(i, j)=\frac{\frac{C(i, j)}{\sum_{i, j} C(i, j)}}{\left(\frac{C(i)}{\sum_{k} C(k)}\right)\left(\frac{C(j)}{\sum_{k} C(k)}\right)}
$$

where $C(i, j)$ is the number of gene product that is annotated with both term $i$ ) and term $i), C(i)$ and $C(j)$ are the number of gene product that is annotated with term $i$ ), term $i$ ) respectively, and $C(k)$ is total number of gene product.

Table 2.1: Similarity measures and their mixing strategy found in literature

$\begin{array}{lll}\text { Similarity measures } & \text { Strategy } & \text { Ref } \\ \text { Lord } & \text { AVG } & \text { Lord et al. (2003) } \\ \text { Dice } & \text { set } & \text { Popescu et al. (2006) } \\ \text { CAS } & \text { RCMAX } & \text { Chitale et al. (2011) } \\ \text { Wang } & \text { BMA } & \text { Chen et al. (2007) } \\ \text { Topoicsim } & \text { RCMAX } & \text { Ehsani \& Drabløs (2016) } \\ \text { RSS } & \text { MAX } & \text { Wu et al. (2006) } \\ \text { simGIC } & \text { groupwise } & \text { Pesquita et al. (2007) }\end{array}$


Having obtained the term's semantic similarity, the next task is to combined the term similarity into gene similarity. In gene annotation, a gene can be annotated with multiple GO term. Calculating gene similarity based on the GO term thus need to consider multiple terms in each gene. There are several different strategies available for this purposes. Table 2.1 summarize some strategies that are used by different similarity measures to transform term similarity to gene similarity. The strategies are:

Average (AVG) strategy calculates the average similarity over all pair of terms of gene products being compared.The average strategy is used by Lord measure. It is defined as:

$$
S\left(g_{i}, g_{j}\right)=\frac{1}{m * n} \sum_{i=1}^{n} \sum_{j=1}^{m}\left(S\left(t_{i}, t_{j}\right)\right)
$$

where $n$ and $m$ are the number of terms in gene $i$ and $j$ respectively.

Maximum (MAX) strategy uses the maximum similarity of pair of terms from two gene products as gene product similarity measure. The maximum strategy is used by RSS measure. It is define as:

$$
S\left(g_{i}, g_{j}\right)=\max _{1 \leq i \leq n, 1 \leq j \leq m}\left(S\left(t_{i}, t_{j}\right)\right)
$$

rcmax (RCMAX) strategy uses matrix of gene terms. It uses the maximum score from the average of maximum value for each column and each row.This strategy is used by TopoICSIM and CAS measures. It is define as:

$$
S\left(g_{i}, g_{j}\right)=\max \left(\frac{\sum_{i=1}^{n} \max _{j} S\left(t_{i}, t_{j}\right)}{n}, \frac{\sum_{j=1}^{m} \max _{i} S\left(t_{i}, t_{j}\right)}{m}\right)
$$

Best match average (BMA) strategy also uses matrix of gene terms. It calculates the average of maximum term similarity value from each row and each columns. 
TheBMA strategy is used by Wang measure. It is define as:

$$
S\left(g_{i}, g_{j}\right)=\frac{\left(\sum_{i=1}^{m} \max S\left(t_{i}, t_{j}\right)+\sum_{j=1}^{n} \max S\left(t_{i}, t_{j}\right)\right)}{m+n}
$$

Unlike previously described pairwise approach, measures that uses groupwise approach do not need to perform two step calculations. For example, simGIC (Pesquita et al. (2007)) used set operation on calculating the semantic similarity of two genes. However, instead of counting the number of term occurrences in both genes, It sums the IC of term $t$. It is formally defined as:

$$
S\left(g_{i}, g_{j}\right)=\frac{\sum_{t \in g_{i} \cap g_{j}} I C(t)}{\sum_{t \in g_{i} \cup g_{j}} I C(t)}
$$

Other pairwise measures consider the term as a vector and calculate the semantic similarity of two genes by using vector operation such as cosine. Gan (Gan (2014)) represented gene as a vector of IC. The semantic similarity of two genes than is calculated by cosine similarity as:

$$
S\left(g_{i}, g_{j}\right)=\frac{\sum_{1 \leq i \leq|V|} x_{g_{i}} x_{h_{i}}}{\sqrt{\sum_{1 \leq i \leq|V|} x_{h_{i}}^{2}}}
$$




\section{Chapter 3}

\section{DATA AND METHOD}

\subsection{The GO dataset and gene annotation}

The GO term and the Gene Ontology Annotation (GOA) version 2018-10-01 were downloaded from the Gene Ontology Consortium, http://archive.geneontology.org/. This GO version contains $31024 \mathrm{BP}, 11940 \mathrm{MF}$, and $4379 \mathrm{CC}$ terms. We used human GOA to associate between the gene products and GO terms. There are 469,828 association of 19678 proteins were present in the human GOA dataset (390389 associations of 18084 proteins without IEA annotation).

\section{$3.2 \quad$ UniProt dataset}

We used the human UniProt identifier mapping (version 9606) to map the identifier used in KEGG dataset and BioGrid dataset.

\subsection{The KEGG pathway database}

We downloaded human KEGG pathway data set from Kyoto Encyclopedia of Genes and Genomes database (Kanehisa \& Goto (2000)), http://rest.kegg.jp. The database consists of 320 pathways, and the number of gene in the pathways range from 1 to 448 .

We evaluated our semantic similarity measure against other measures by predicting the genes membership in metabolic pathways. The assumption is that the genes that belong to the same pathways are more similar to each other since they share similar function (Ehsani \& Drabløs (2016)). 
To predict whether a gene belongs to certain metabolic pathways, functional relatedness. We generated positive and negative datasets for each pathway in KEGG pathway.

For a given pathway, we build a classifier and test its predictive power by 10-fold cross validation. Specifically, all genes that belong to the pathway make up a positive dataset, whereas a negative dataset is generated by choosing randomly from genes of all other pathways in KEGG database. We then randomly divided the positive dataset into ten subsets. Each subset is reserved as a positive test set, and the remaining nine subsets are merged into a positive train set. This process is repeated 10 times to ensure each subset is used once as test. For a classifier based on supervised learning, a negative train set and a negative test set are prepared in a similar manner as their positive counterparts. The performance of a classifier is evaluated on the test set and averaged over 10 fold cross-validation.

\subsection{Gene representation}

In order to calculate semantic similarity between two genes (or their corresponding proteins as we hereafter use gene and protein with nodistinction), we adopt the vector space model (VSM) approach, which is commonly used in document information retrieval. In this model, each document is represented by vector of words or keywords. Here, we represented the gene as vector of GO terms. We build the vector of genes by using GO terms as the gene's features. While a simplistic approach is a binary vector - with 1 or 0 representing the presence or absence of GO terms in annotation of the given gene, our method considers both of the structure of the GO graph and the information content of the GO terms in building the vector of the gene and their corresponding annotations.

Specifically, before we build the gene vector, we first calculated the semantic

value $(\mathrm{SV})$ for each GO term in the annotation of a given gene. We calculated the SV as the information content of GO term. 
We used a normalized information content by dividing the information content of the term $t_{i}$ with the maximum IC in whole set of GO terms $T$ as follows:

$$
I C_{n}\left(t_{i}\right)=\frac{I C\left(t_{i}\right)}{\max _{t_{j} \in T} I C\left(t_{j}\right)}
$$

Then we include the ancestor terms of original annotation in a given gene to build gene vector: for each annotation term $t$ in a given gene, we assigned the weighted semantic value for all its ancestor terms $t_{p}$, defined as follows.

$$
S V\left(t_{p}\right)=w^{d_{p}} I C_{n}\left(t_{p}\right)
$$

where $w$ is the weight, in this case we use a fix constant of 0.5 , and $d_{p}$ is the path length of the ancestor term $p$ to the annotation term $t$. The path length is defined as the difference of the maximum depth between the two terms, i.e. between term $t_{i}$ and its ancestor.

When there are multiple GO terms in the annotation of a given gene, it is possible that these GO terms may have ancestor terms in common. Therefore, during inclusion of the ancestor terms, for each annotation term in a given gene, a common ancestor term will have multiple semantic values, each for a descendant in the original annotation as the common ancestor term may receive a semantic value from all of its descendants differently. Hence, we calculated the average of these values as the new semantic values for a common ancestor term $t_{p}$.

After this procedure, a gene is represented as a vector of n-dimension, where $n=|T|$, each dimension corresponding to one GO term in the gene ontology hierarchy, with a semantic value being either a) the normalized information content if the GO term is present in the gene annotation, or b) a value assigned as above for a GO term whose descendant(s) is present, or c) a value of zero if a GO term is not of either of the two former cases.

The similarity score of two genes $\mathrm{q}$ and $\mathrm{p}$ in is then defined as dot product of 
their corresponding vector as

$$
s(q, p)=\sum_{t \in T} S V_{q}(t) * S V_{p}(t)
$$

\subsection{Pathway membership prediction}

As mentioned in Introduction, our method brings together the two perspectives - incomplete metabolic pathway and GO annotation of individual proteins - to infer whether a query protein is a member of the incomplete pathway. While there exist some more sophisticated methods that construct a model for the pathway to integrate and leverage various information, our method simply use the set of proteins of the incomplete pathway $P$ as a "model" and calculate a score $S(q, P)$ for a query protein $q$ to determine whether $q$ belong to the model, i.e., $q$ as a member of the pathway $P$. For example, if $S(q, P)$ is higher than a threshold, $q$ is predicted as a member, otherwise not a member. In this work, instead of using a specific threshold, a ROC score is used to evaluate the prediction performance.

The score $S(q, P)$ is the average similarity between the query protein and the proteins that are already known to be in the pathway $P$. Specifically,

$$
S(q, P)=\sum_{p \in P} S(q, p) /|P|
$$

where $s(q, p)$ is the similarity score between query protein q and a member protein $\mathrm{p}$ as calculated by 3.3 and $|P|$ is the number of known proteins for the incomplete pathway $\mathrm{P}$.

\subsection{Performance evaluation}

We used receiving operating characteristic (ROC) curve analysis to evaluate the performance of our method. ROC curve measure the performance of classifier at 
various threshold setting, and represents the trade off between true positive rate (TPR) and false positive rate (FPR). The TPR and FPR are defined as:

$$
F P R=\frac{F P}{(F P+T N)} T P R=\frac{T P}{(T P+F N)}
$$

where $F P, T N, T P$, and $F N$ are the number of false positive, true negative, true positive and false positive respectively. The ROC curve of perfect classifier have the area under the ROC curve (AUC) of 1 . The perfect curve rise steeply from bottom left to top left and move toward top right. The AUC is calculated using trapezoidal rule as:

$$
A U C=\frac{1}{2} \sum_{k=1}^{n}\left(X_{k}-X_{k-1}\right)\left(Y_{k}-Y_{k-1}\right)
$$




\section{Chapter 4}

\section{RESULTS AND DISCUSSIONS}

\subsection{Random gene pair semantic similarity distribution}

Biologically, functionally similar genes are far less common than those that are functionally dissimilar. Thus, a set of randomly pair of genes should have semantic similarity score close to zero (Gan (2014). A good semantic similarity measures should reflect this, and should not overestimate the semantic similarity of set of random pairs, i.e. have semantic similarity score away from zero for set of random pairs. We follow the same approach as Gan (2014) to calculate the distribution of semantic similarity score of set of random pair of genes. We generated 100000 random pairs, and calculate the average score of the semantic similarity of the random pairs.

Table 4.1 shows the distribution of semantic similarity average score of random pairs for different methods. When comparing the average score across three ontologies, in most of the measures, $\mathrm{BP}$ ontology produces the minimum average score while CC ontology produces the maximum average score. For example, the average score of random pair combination of TopoICSim measure is lowest in BP (0.2), but for Lord Measures the lowest average score is in CC ontology (0.031). The highest scores in $\mathrm{CC}$ ontology for most measures might indicate that many of the random pairs produce high semantic similarity score. This is reasonable since the number of term in CC ontology is the lowest among three ontology. Thus the probability of two pairs of gene share common terms in $\mathrm{CC}$ are higher than in $\mathrm{BP}$ and $\mathrm{MF}$. As a result the average similarity score is higher than BP and MF. The number of GO terms in BP, MF, and CC ontologies are 31024, 11940, and 4379 respectively. 
Table 4.1: The average similarity score of 10000 random pair genes

$\begin{array}{llll}\text { Methods } & \text { BP } & \text { CC } & \text { MF } \\ \text { TopoICSim } & 0.200 & 0.432 & 0.386 \\ \text { Lord } & 0.043 & 0.031 & 0.047 \\ \text { Wang } & 0.329 & 0.594 & 0.565 \\ \text { RSS } & 0.463 & 0.612 & 0.556 \\ \text { CAS } & 0.005 & 0.001 & 0.002 \\ \text { simGIC } & 0.003 & 0.050 & 0.060 \\ \text { cos-bsc } & 0.007 & 0.107 & 0.155 \\ \text { GAN } & 0.005 & 0.075 & 0.075 \\ \text { our } & 0.009 & 0.092 & 0.074\end{array}$

\subsection{Metabolic pathway membership prediction}

We evaluate the performance of our method in predicting the membership of genes in KEGG metabolic pathway. The main idea behind this is that the genes in the same metabolic pathway are performing more similar biological process than the genes in different pathways (Ehsani \& Drabløs (2016)).

We compare the performance of our method against other methods by using ROC analysis. Table 4.2 and figure 4.1 show ROC score and ROC curve for pathway membership prediction using our method, in comparison to using a suite of different ontology-based gene similarity methods mentioned in the Related Works. Because GO has three separate hierarchies: BP, CC, and MF, we evaluate prediction performance for each hierarchy. In general, the best result are obtained for all methods when BP ontology is used in calculating gene similarity, followed by either CC or MF depends on the methods used. This pattern is consistent with our intuition as metabolic pathways are better characterized as biological processes (BP). It is clear that our method achieves the best performance with a ROC score of 0.937, from using BP ontology. However, for CC and MF ontology, our method is not the best performance.

Since the prediction accuracy of our method is high, it is interesting to see whether our method is applicable in predicting the gene membership in the pathways for gene or gene product that has no annotations, or in other word for predicting the 
membership of unknown gene or gene products. However, our method only applicable when the annotations are available for the gene. When calculating the semantic similarity between two genes, if the query gene vector is zero vector, then the cosine similarity for this pair will be zero. The query gene then will be categorized as not a member of metabolic pathway in question.

Table 4.2: The area under the curve ROC of semantic similarity measure for BP, MF and CC ontology

$\begin{array}{llll}\text { Methods } & \text { BP } & \text { CC } & \text { MF } \\ \text { TopoICSim } & 0.869 & 0.715 & 0.719 \\ \text { Lord } & 0.848 & 0.688 & 0.803 \\ \text { Wang } & 0.867 & 0.729 & 0.725 \\ \text { RSS } & 0.864 & 0.713 & 0.716 \\ \text { CAS } & 0.910 & 0.795 & 0.856 \\ \text { simGIC } & 0.922 & 0.795 & 0.746 \\ \text { cos-bsc } & 0.914 & 0.774 & 0.656 \\ \text { Gan } & 0.922 & 0.783 & 0.774 \\ \text { our } & 0.937 & 0.783 & 0.821\end{array}$


Figure 4.1: ROC curve plot for gene membership prediction

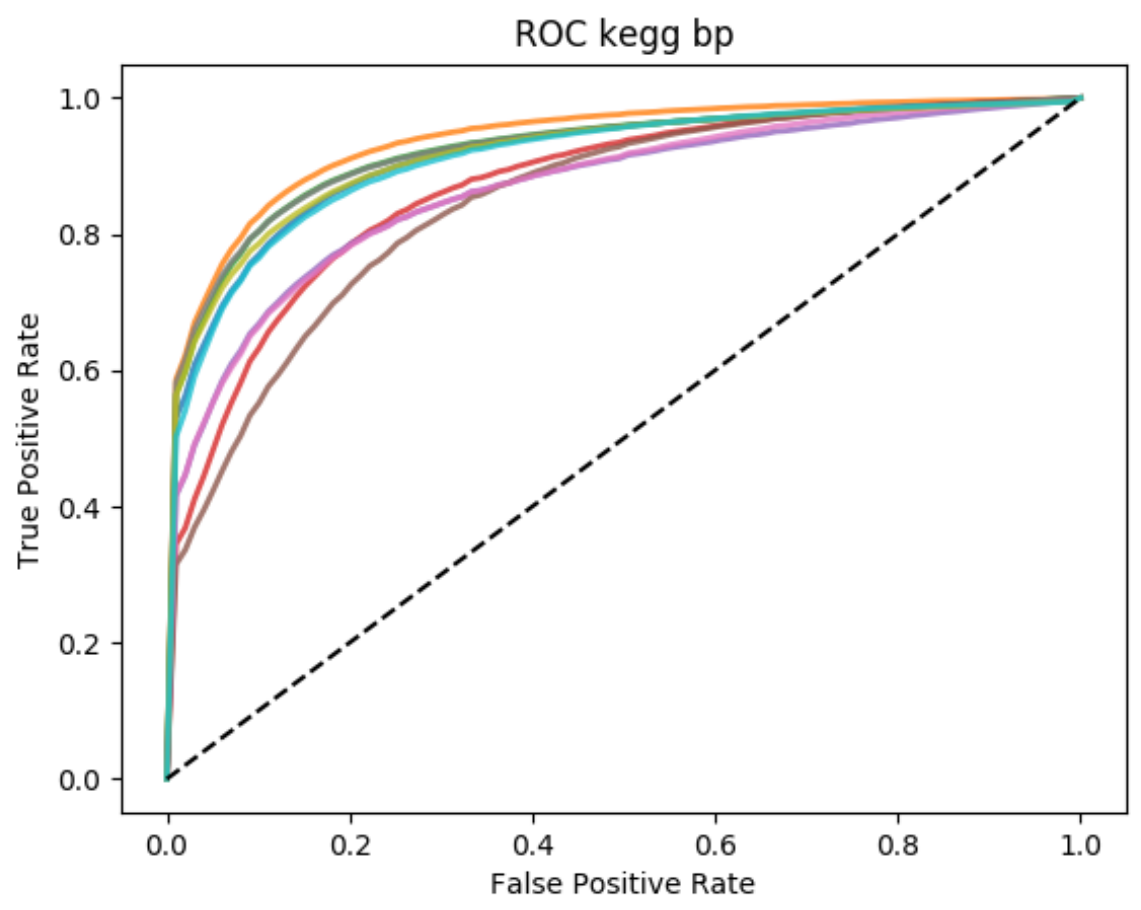

(a)

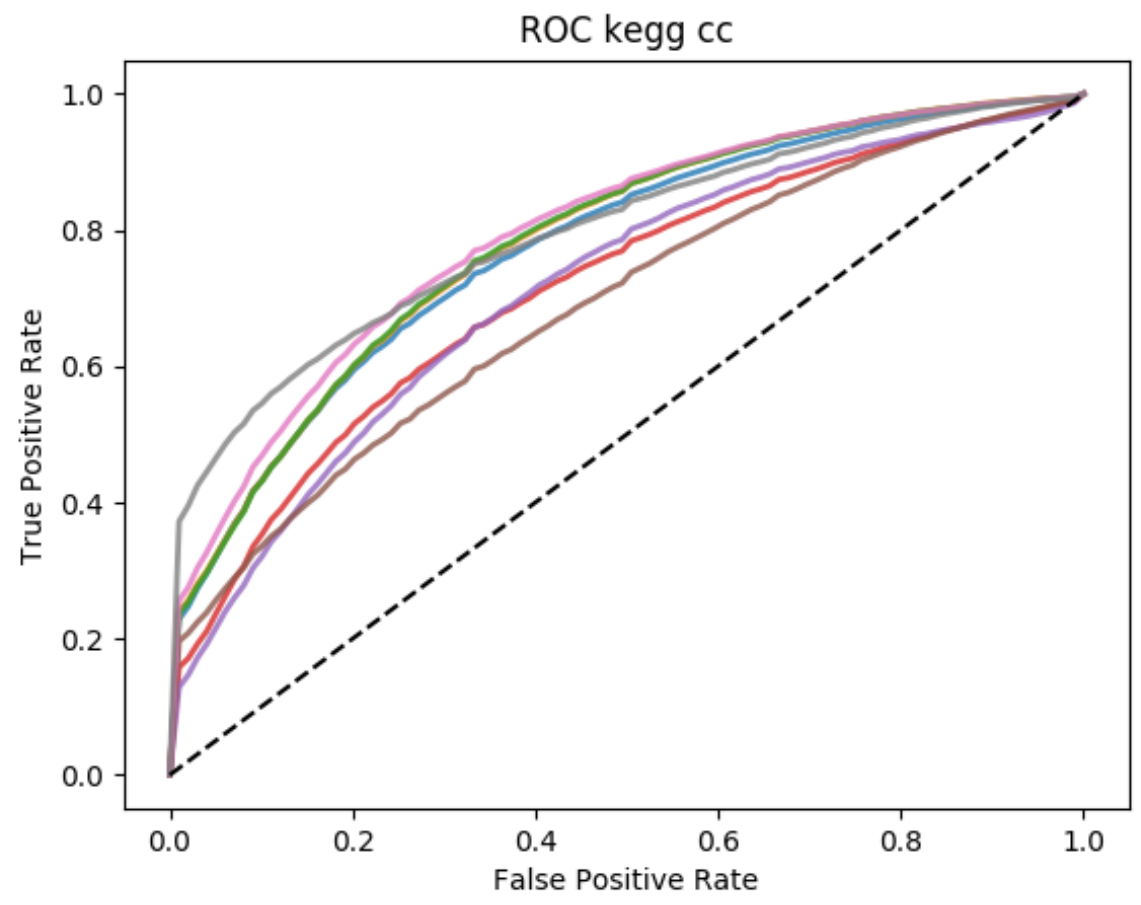

(b) 
Figure 4.1: continued

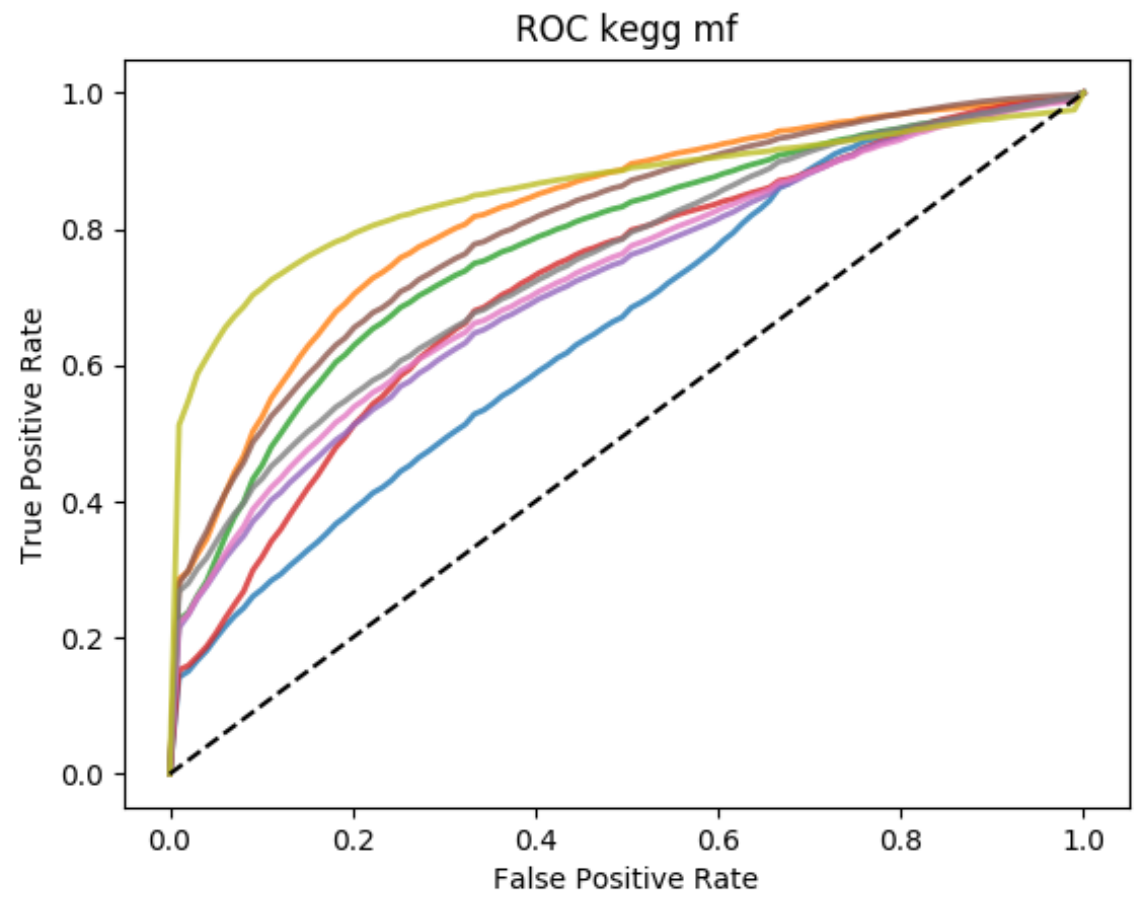

(a)

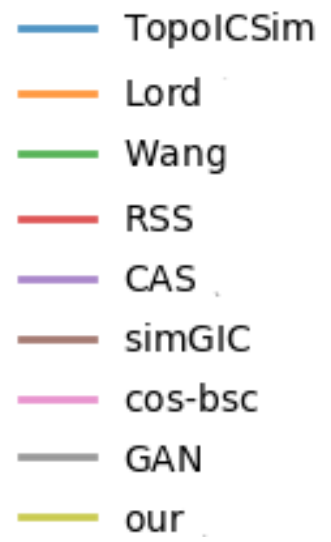

ROC curve plot of KEGG genes membership prediction for (a) BP (b) MF, and (c) CC ontology 
We then further evaluated the improvement of our measure against other methods. Table 4.3 shows that compared to other methods along with p-value from t-test on the 10-fold cross-validation results, our method can achieve better performance than most of measures in all ontologies. for example, our method outperformed other measures up to $11 \%$ when it is compared against Lord method. Similarly, in CC and MF ontologies our measure can outperform almost up to $14 \%$ (comparison against Lord in $\mathrm{CC}$ ontology) and 25\% (comparison against cosine basic) respectively. However, our measures unable to outperform the CAS in both CC and MF ontology, as well as simGIC and GAN in CC ontology. The p-value score also shows that almost all differences are statistically significant ( $\mathrm{p}$ value less than 0.05 ) with exception in CC ontology where there is no statistically significant improvement between our method and Gan.

Table 4.3: Improvement of our measure against other similarity measures in $\mathrm{BP}, \mathrm{CC}$ and MF ontology (in percentage)

\begin{tabular}{lrlrlrl} 
Methods & \multicolumn{1}{c}{ BP } & p-value & CC & p-value & \multicolumn{1}{c}{ MF } & p-value \\
TopoICSim & 7.800 & $<2 \mathrm{e}-16$ & 9.505 & $<2 \mathrm{e}-16$ & 14.145 & $<2 \mathrm{e}-16$ \\
Lord & 10.559 & $<2 \mathrm{e}-16$ & 13.792 & $<2 \mathrm{e}-16$ & 2.261 & $<2 \mathrm{e}-16$ \\
Wang & 8.141 & $<2 \mathrm{e}-16$ & 7.316 & $<2 \mathrm{e}-16$ & 13.165 & $<2 \mathrm{e}-16$ \\
RSS & 8.438 & $<2 \mathrm{e}-16$ & 9.718 & $<2 \mathrm{e}-16$ & 14.570 & $<2 \mathrm{e}-16$ \\
CAS & 3.013 & $<2 \mathrm{e}-16$ & -1.575 & $<2 \mathrm{e}-16$ & -4.169 & $<2 \mathrm{e}-16$ \\
simGIC & 1.636 & $<2 \mathrm{e}-16$ & -1.531 & $<2 \mathrm{e}-16$ & 10.075 & $<2 \mathrm{e}-16$ \\
cos-bsc & 2.563 & $<2 \mathrm{e}-16$ & 1.189 & $4.27 \mathrm{E}-05$ & 25.207 & $<2 \mathrm{e}-16$ \\
Gan & 1.671 & $<2 \mathrm{e}-16$ & -0.030 & 0.915 & 5.992 & $<2 \mathrm{e}-16$
\end{tabular}

While many ontology-based similarity methods evaluate their performance by predicting gene products membership in metabolic pathway, typically they only choose a subset of the metabolic pathways from KEGG database. For example, TopoICSim selected only 13 metabolic pathways with the number of genes in each pathway fewer than 40. In this project, we evaluated the performance of pathway membership prediction for all metabolic pathways in KEGG database, and we further evaluate the performance with different set of pathways based on the number genes in the pathways. Specifically, we divided the pathways into three groups, 1) pathways that contain 
less than 100 genes, 2) pathways that contain between 101 to 200 genes, and 3) pathway that contain more than 200 genes.

Table 4.4 shows that in general, as the number of genes in the pathway increase, the performance of the methods decrease. Most methods perform best in pathways that contain less than 100 genes. For example in BP ontology, the ROC scores of methods in the less than 100 genes pathways ranges between .873 (Lord) to .947 (our method), while in pathways that contain 101 to 200 genes and more than 200 genes the ROC scores range between .820 (TopoICSim) to .919 (our method) and .782 (Lord) to .903 (our method) respectively. In comparison among three ontologies, most of the measures perform best when using BP ontology. For example, in the group of pathways that contain less than 100 genes, the ROC scores of BP, CC and MF are ranging between .873 (Lord) to .947 (our method), .720 (RSS) to .807 (our method) and .664 (cos-basic) to .830 (Lord and our method) respectively. In BP ontology, our measure outperformed other measures for all pathway groups. It is clearly seen in the table that our method is one of the top performances in all categories. 
Table 4.4: Performance of measures in predicting genes membership of metabolic pathways that contains different number of genes.

\begin{tabular}{l|ccc|ccc|ccc} 
& \multicolumn{3}{|c|}{ BP } & \multicolumn{3}{c|}{ CC } & \multicolumn{3}{c}{ MF } \\
\hline Methods & $<100$ & $101-200$ & $>200$ & $<100$ & $101-200$ & $>200$ & $<100$ & $101-200$ & $>200$ \\
\hline TopoICSim & 0.893 & 0.820 & 0.816 & 0.738 & 0.666 & 0.653 & 0.731 & 0.687 & 0.728 \\
Lord & 0.873 & 0.794 & 0.782 & 0.723 & 0.617 & 0.583 & 0.830 & 0.741 & 0.760 \\
Wang & 0.886 & 0.823 & 0.840 & 0.744 & 0.696 & 0.712 & 0.750 & 0.665 & 0.701 \\
RSS & 0.883 & 0.823 & 0.833 & 0.720 & 0.699 & 0.701 & 0.731 & 0.679 & 0.712 \\
CAS & 0.922 & 0.888 & 0.867 & 0.801 & 0.783 & 0.776 & 0.846 & 0.879 & 0.881 \\
simGIC & 0.926 & 0.917 & 0.902 & 0.816 & 0.752 & 0.740 & 0.753 & 0.732 & 0.722 \\
cos-bsc & 0.920 & 0.904 & 0.884 & 0.794 & 0.729 & 0.725 & 0.664 & 0.637 & 0.636 \\
GAN & 0.926 & 0.916 & 0.900 & 0.806 & 0.736 & 0.719 & 0.776 & 0.772 & 0.764 \\
our & 0.947 & 0.919 & 0.903 & 0.807 & 0.734 & 0.715 & 0.830 & 0.801 & 0.799 \\
\hline
\end{tabular}


Since genes in the same metabolic pathways are assumed to have similar biological process, we examined this by exploring the mean value of pairwise semantic similarity score between genes in the pathway (inside-inside genes) as well as the semantic similarity score between genes outside the pathway and genes inside the pathway (outside-inside genes). We reasoned that the inside-inside genes should have a higher similarity score than the similarity score of inside-outside genes since the inside-inside genes are more similar to each other than to the outside genes. Therefore, we can calculate the ratio of similarity scores of inside-inside genes against inside-outside genes to check whether the assumption holds. The ratio should be bigger than 1 if the inside-inside genes are more similar to each other than to outside genes.

Table 4.5 show the ratio of semantic similarity scores between inside-inside genes to inside-outside genes. All methods show the ratio bigger than 1, which indicates that the genes inside the pathway have higher similarity scores between them. Amongst the method being compared, CAS shows the highest ratio for all ontologies.

Table 4.5: The ratio of semantic similarity scores

$\begin{array}{llll}\text { Methods } & \text { BP } & \text { CC } & \text { MF } \\ \text { TopoICSim } & 1.666 & 1.201 & 1.362 \\ \text { Lord } & 1.746 & 1.341 & 1.942 \\ \text { Wang } & 1.329 & 1.138 & 1.101 \\ \text { RSS } & 1.254 & 1.140 & 1.207 \\ \text { CAS } & 3.672 & 3.892 & 7.828 \\ \text { simGIC } & 2.894 & 1.301 & 1.337 \\ \text { cos-bsc } & 2.636 & 1.230 & 1.203 \\ \text { GAN } & 2.636 & 1.230 & 1.203 \\ \text { our } & 2.271 & 1.222 & 1.459\end{array}$

Other semantic similarity based methods, such as those are mainly based on the structure of the graph, are limited to use only one of the three semantic similarities. The example of those methods are RSS and CAS. These methods calculate the similarity by including the structure of the graph. Since gene ontology consists a separate three ontologies, i.e. $\mathrm{BP}, \mathrm{MF}$ and $\mathrm{CC}$ ontologies, each with its own tree structure and root node, combining all three ontologies to calculate the semantic similarity of two genes is 
difficult. Our method on the other hand are based on vector. Each gene is represented as a vector of GO terms. If we want to use the three ontologies, the simplest approach is by combining the tree ontologies into a single vector to represent a gene. Table 4.6 show the ROC score for combine ontologies in 3 methods. The ROC scores of combine ontologies are lower than the score for BP ontology, however higher than the other two ontologies in three methods. For example, the ROC score of our method by using combined ontologies are .933 which is lower than ROC score for BP ontology (.937) but higher than ROC score for CC (.783) and MF (.821) ontologies. The difference of ROC score between BP ontology only and three ontologies, however, are very small, for cosbsc, GAN and our methods, the differences are 0.074, 0.002, and 0.004 respectively. This pattern still consistent with our intuition that metabolic pathways are better characterized by BP ontology than other ontologies, although the application of all ontologies may be consider to represent the gene better as it includes all terms in three ontologies.

Table 4.6: ROC score for combine ontologies

$\begin{array}{lc}\text { Method } & \text { AUC } \\ \text { cos-bsc } & 0.840 \\ \text { GAN } & 0.920 \\ \text { our } & 0.933\end{array}$

We also compare the performance of our method by measuring the running time. For this purpose, we randomly chose 4 pathways, i.e hsa00072, hsa00532, hsa04979, hsa05169, of different sizes that contain 10, 20, 50, and 200 genes respectively. Table 4.7 shows that the running time of our method outperformed others. Methods that based on pairwise approach, i.e calculating pairwise semantic similarity of two terms between two pairs of genes, such as Lord, RSS or CAS are fast when the number of the genes are small. The time complexity of calculating the gene similarity in these methods are quadratic $O(n m)$, where $\mathrm{n}$ and $\mathrm{m}$ are the number of terms of the two genes. Calculating the similarity of genes in the pathways are not problem when the number of gene members in the pathway are small. However, it will need considerably more time 
to calculate the semantic similarity when the number of gene members of the pathway are big. In addition, some methods, especially methods that used the structure of the graph such as RSS, calculating of pair of terms depends on the complexity of the tree. The time complexity of calculating the similarity of the terms is linear with the depth of the tree or the distance between the two terms is logarithmic $O(n \log n)$ where $\mathrm{n}$ is the depth of the graph or the length between the two terms depending what approach used in the methods.

On the contrary, vector based approach only need to build the gene vector once. Once the vector is build, the similarity between the two genes can be calculated directly by vector operation. Thus for this kind of methods, the time complexity is linear $O(n)$ where $\mathrm{n}$ is the number of the pair of genes.

Thus when comparing the running time for different methods, we can see clearly in table 4.7 that our method outperformed other methods. When compare to other vector based method, such as GAN, the running time is equal. Out method running time is linear as the number of pathway size, however other methods, especially the pairwise based method time requirement increase drastically when the pathways size getting bigger.

Table 4.7: Running time of similarity measures in seconds

$\begin{array}{lccccr}\text { Methods } & \text { hsa00072(10) } & \text { hsa00532(20) } & \text { hsa04979(50) } & \text { hsa05169(200) } & \text { total } \\ \text { TopoICSim } & 9.80 & 10.65 & 296.68 & 3734.78 & 4051.91 \\ \text { Resnik } & 0.06 & 0.27 & 7.89 & 2119.36 & 2127.57 \\ \text { Wang } & 0.05 & 0.25 & 8.64 & 1685.79 & 1694.73 \\ \text { RSS } & 119.69 & 547.79 & 9446.41 & 71544.76 & 81658.65 \\ \text { CAS } & 218.82 & 309.55 & 2313.55 & 11126.51 & 13968.43 \\ \text { simGIC } & 0.71 & 1.67 & 5.36 & 106.56 & 114.31 \\ \text { cos basic } & 0.79 & 0.85 & 1.45 & 3.12 & 6.21 \\ \text { cos } & 0.63 & 1.43 & 1.99 & 4.44 & 8.49 \\ \text { cos ic } & 0.57 & 0.71 & 1.54 & 3.67 & 6.49\end{array}$

We calculated the running time on Linux machine with Intel Xeon ES-2680 2.7GHz and $32 \mathrm{~GB}$ of RAM. 


\subsection{Distribution of high semantic similarity score}

Our method applies cosine similarity to determine the similarity between 2 genes which relies on semantic terms, i.e GO terms. If two genes have the same annotations, the similarity score of the two genes are 1. Although semantically the two genes are similar, the two genes may not always be member of the same pathway. Therefore, we explore the distribution of the similarity score of all possible gene pairs in the BP ontology in regard to the gene membership to the pathways. For each pair of genes,

the possibilities of the genes in regard of the member of the pathways are: (1) both of the genes are member of the pathway(s) whether they are belong to the same pathway or different pathways (in-pathway), (2) or not member of any pathway (out-pathway), or (3) only one of the genes are belong to pathway(s) (in-out-pathway).

Table 4.8 shows the number of the pairs in those three categories. It shows that most of the pairs have both genes that are not member of any pathway, except where the similarity score are 1 or score between .1 and .2. This is as expected since the total number of the genes in the KEGG pathway are 7453 genes, which is only $12 \%$ of the total human genes listed in NCBI. However, for low semantic similarity score, especially score between 0.1 to 0.2 , the number of pairs that both of the genes belong to pathways are bigger than any other type of pairs. In general, the number of both genes not belong to any pathway increases as the similarity score increases, while the number of pairs that the two genes or any one of the genes belong to pathway decrease as the similarity score increase.

For measuring the performance of our method, we used ROC analysis which based on ranking of the semantic similarity score. If the two genes being compared have high similarity score, the pairs will place itself in the higher ranking, and will be predicted to be member of the same pathway. For pairs that have semantic similarity score of 1, the in-pathway pairs are bigger than any other pairs categories. However, for most of the pairs in other range of semantic similarity, the number of the pairs that both of the genes are not the member of the pathway are the biggest.

For gene pairs that have identical annotation, it is likely that the two genes are 
Table 4.8: Total number of pairs in regard of their gene's member status in the pathway

$\begin{array}{lllll}\text { Similarity score } & \text { in-out } & \text { in } & \text { out } & \text { Total } \\ \operatorname{sim}=1 & 1631(5.6) & 19337(66.1) & 8272(28.3) & 29240 \\ .9 \leq \operatorname{sim}<1 & 3024(3.6) & 2512(3) & 78664(93.4) & 84200 \\ .8 \leq \operatorname{sim}<.9 & 3302(6.8) & 2637(5.4) & 42911(87.8) & 48850 \\ .7 \leq \operatorname{sim}<0.8 & 4372(5.3) & 30971(37.7) & 46916(57) & 82259 \\ .6 \leq \operatorname{sim}<.7 & 17749(16.8) & 8708(8.2) & 79452(75) & 105909 \\ .5 \leq \operatorname{sim}<.6 & 24047(17.2) & 16559(11.8) & 99314(71) & 139920 \\ .4 \leq \operatorname{sim}<.5 & 54867(22.4) & 20114(8.2) & 170184(69.4) & 245165 \\ .3 \leq \operatorname{sim}<.4 & 135260(30.4) & 30412(6.8) & 279715(62.8) & 445387 \\ .2 \leq \operatorname{sim}<.3 & 361801(38.5) & 83528(8.9) & 495606(52.7) & 940935 \\ .1 \leq \operatorname{sim}<.2 & 1211831(46.3) & 416528(15.9) & 988772(37.8) & 2617131\end{array}$

actually synonym genes,i.e. genes with multiple names, or belong to the same family member. Gene families are group of genes that are similar in nucleotide sequences or amino acid sequences and they also share similar cellular functions. These genes are commonly arise from genome duplication events (Harris \& Hofmann (2015)). Where synonym genes refer to the same gene, genes in gene families may be different genes. Therefore, we try to map the genes in each pair to whether they are synonym or member of the same gene families. We found that the percentage of pair of genes belong to the same family member is quite high in all semantic similarity score, even for low semantic similarity score. The percentage for all score range are above $60 \%$ (figure 4.2). This indicates that the annotation for genes in the family genes can be very different, although it is widely accepted that family genes have similar cellular functions. 
Figure 4.2: Distribution of gene family, interacting, and synonym genes in the pairs

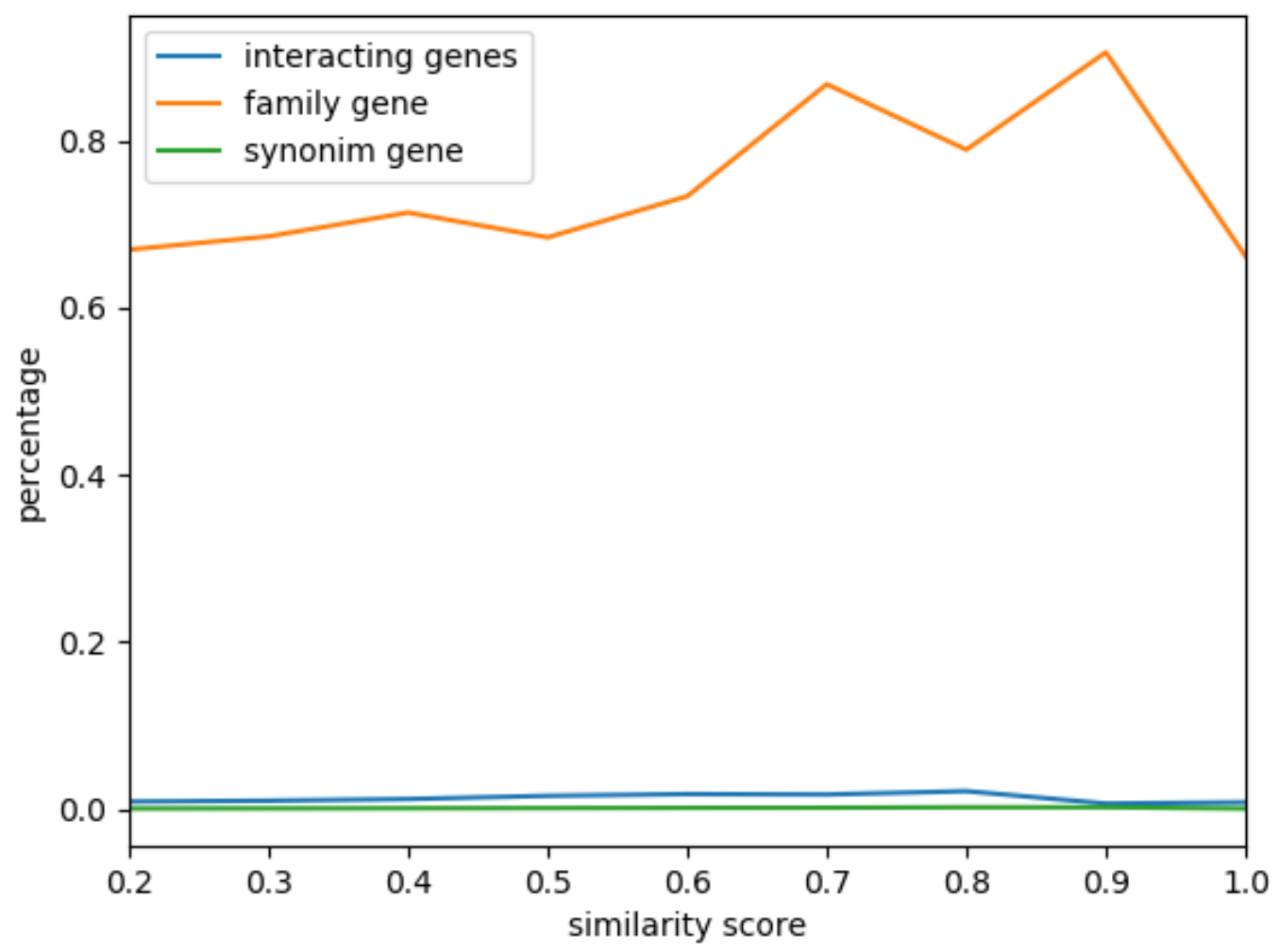

(c)

Percentage of pairs that both genes in the pair are the same family, are interacting genes, or are synonim genes 


\section{Chapter 5 CONCLUSION}

Metabolic pathway is important in understanding the underlying mechanisms in the biological process and celullar functions of cell. Predicting the membership of the gene to pathways in which the gene participate can greatly help to understand the gene function in the pathways. In this work, we developed a method for pathway membership inference based gene ontology (GO) similarity between a query protein and proteins that are known to the members of a given pathway. We use human metabolic pathway and human gene annotation dataset. We demonstrated that, by replacing binary vector of GO term annotation for a gene with the information content of individual GO terms and incorporating GO hierarchy with ancestor nodes that are directly present in gene annotation, we can create information rich vector representation for a gene, which enables accurate pathway membership prediction even with a simple classifier based just on positive training example and cosine similarity, outperforming some more sophisticated methods both by prediction accuracy and running time. In our test, the performance of our method outperformed other methods when using BP ontology. While other methods that rely on the structure of the graph use the three ontologies separately since the three ontologies are separate graph each with its own root node, our method can integrate the three ontologies easily. The performance of our method when using combine ontology smaller than when BP ontology use alone but higher then when using $\mathrm{CC}$ or MF ontologies. These result indicates that $\mathrm{BP}$ ontology is better characterize metabolic pathways than other ontology.

As our method only use a discriminative classifier that calculate the similarity between the set of proteins in incomplete pathway and query pathway, as future work use of a discriminative classifier that can take advantage of both positive and negative 
training examples, such as support vector machines, will be explored. In addition, the use of dataset other than human may be interesting to be explored to see if the same result can be generalized. Another interesting future direction is to develop method to infer unannotated gene products membership in metabolic pathways since our method has limitation to only able to predict gene membership in the pathway only for genes that has GO annotation. 


\section{REFERENCES}

Al-Mubaid, H. \& Nagar, A. (2008), 'Comparison of four similarity measures based on go annotations for gene clustering', 2008 IEEE Symposium on Computers and Communications pp. $531-536$.

Brown, D. G., Rao, S., Weir, T. L., O’Malia, J., Bazan, M., Brown, R. J. \& Ryan, E. P. (2016), 'Metabolomics and metabolic pathway networks from human colorectal cancers, adjacent mucosa, and stool', Cancer \&3 Metabolism 4(1), 11.

URL: https://doi.org/10.1186/s40170-016-0151-y

Cameron, S. J., Lewis, K. E., Beckmann, M., Allison, G. G., Ghosal, R., Lewis, P. D. \& Mur, L. A. (2016), 'The metabolomic detection of lung cancer biomarkers in sputum', Lung Cancer 94, 88 - 95.

URL: http://www.sciencedirect.com/science/article/pii/S0169500216302252

Chen, C.-F., Wang, J. Z., Yu, P. S., Payattakool, R. \& Du, Z. (2007), 'A new method to measure the semantic similarity of go terms', Bioinformatics 23(10), 1274-1281. URL: https://dx.doi.org/10.1093/bioinformatics/btm087

Chitale, M., Palakodety, S. \& Kihara, D. (2011), 'Quantification of protein group coherence and pathway assignment using functional association', BMC bioinformatics 12, 373-373.

URL: https://www.ncbi.nlm.nih.gov/pubmed/21929787

Duan, Z.-H., Hughes, B., Reichel, L., Perez, D. M. \& Shi, T. (2006), 'The relationship between protein sequences and their gene ontology functions', BMC Bioinformatics $7(4)$, S11.

URL: https://doi.org/10.1186/1471-2105-7-S4-S11

Ehsani, R. \& Drabløs, F. (2016), 'Topoicsim: a new semantic similarity measure based on gene ontology', BMC Bioinformatics 17(1), 296.

URL: https://doi.org/10.1186/s12859-016-1160-0

Gan, M. (2014), 'Correlating information contents of gene ontology terms to infer semantic similarity of gene products.', Comput Math Methods Med 2014, 891842.

Guzzi, P. H., Mina, M., Guerra, C. \& Cannataro, M. (2012), 'Semantic similarity analysis of protein data: assessment with biological features and issues.', Brief Bioinform 13(5), 569-585. 
Harris, R. M. \& Hofmann, H. A. (2015), 'Seeing is believing: Dynamic evolution of gene families', Proceedings of the National Academy of Sciences 112(5), 1252-1253. URL: https://www.pnas.org/content/112/5/1252

Jiang, J. J. \& Conrath, D. W. (1997), Semantic similarity based on corpus statistics and lexical taxonomy, in 'Proceedings of the 10th Research on Computational Linguistics International Conference', The Association for Computational Linguistics and Chinese Language Processing (ACLCLP), pp. 19-33.

URL: http://aclweb.org/anthology/097-1002

Kanehisa, M. \& Goto, S. (2000), 'Kegg: kyoto encyclopedia of genes and genomes.', Nucleic Acids Res 28(1), 27-30.

Lin, D. (1998), An information-theoretic definition of similarity, in 'ICML'.

Lord, P. W., Stevens, R. D., Brass, A. \& Goble, C. A. (2003), 'Investigating semantic similarity measures across the gene ontology: the relationship between sequence and annotation.', Bioinformatics 19(10), 1275-1283.

Pesquita, C., Faria, D., Bastos, H. P., Falcão, A. O. \& Couto, F. M. (2007), Evaluating go-based semantic similarity measures.

Popescu, M., M Keller, J. \& Mitchell, J. (2006), 'Fuzzy measures on the gene ontology for gene product similarity', IEEE/ACM transactions on computational biology and bioinformatics / IEEE, ACM 3, 263-74.

Radivojac, P., Clark, W. T., Oron, T. R., Schnoes, A. M., Wittkop, T., Sokolov, A., Graim, K., Funk, C., Verspoor, K., Ben-Hur, A., Pandey, G., Yunes, J. M., Talwalkar, A. S., Repo, S., Souza, M. L., Piovesan, D., Casadio, R., Wang, Z., Cheng, J., Fang, H., Gough, J., Koskinen, P., Toronen, P., Nokso-Koivisto, J., Holm, L., Cozzetto, D., Buchan, D. W. A., Bryson, K., Jones, D. T., Limaye, B., Inamdar, H., Datta, A., Manjari, S. K., Joshi, R., Chitale, M., Kihara, D., Lisewski, A. M., Erdin, S., Venner, E., Lichtarge, O., Rentzsch, R., Yang, H., Romero, A. E., Bhat, P., Paccanaro, A., Hamp, T., Kassner, R., Seemayer, S., Vicedo, E., Schaefer, C., Achten, D., Auer, F., Boehm, A., Braun, T., Hecht, M., Heron, M., Honigschmid, P., Hopf, T. A., Kaufmann, S., Kiening, M., Krompass, D., Landerer, C., Mahlich, Y., Roos, M., Bjorne, J., Salakoski, T., Wong, A., Shatkay, H., Gatzmann, F., Sommer, I., Wass, M. N., Sternberg, M. J. E., Skunca, N., Supek, F., Bosnjak, M., Panov, P., Dzeroski, S., Smuc, T., Kourmpetis, Y. A. I., van Dijk, A. D. J., ter Braak, C. J. F., Zhou, Y., Gong, Q., Dong, X., Tian, W., Falda, M., Fontana, P., Lavezzo, E., Di Camillo, B., Toppo, S., Lan, L., Djuric, N., Guo, Y., Vucetic, S., Bairoch, A., Linial, M., Babbitt, P. C., Brenner, S. E., Orengo, C., Rost, B., Mooney, S. D. \& Friedberg, I. (2013), 'A large-scale evaluation of computational protein function prediction.', Nat Methods 10(3), 221-227. 
Resnik, P. (1999), 'Semantic similarity in a taxonomy: An information-based measure and its application to problems of ambiguity in natural language', J. Artif. Intell. Res. 11, 95-130.

Song, X., Li, L., Srimani, P. K., Yu, P. S. \& Wang, J. Z. (2014), 'Measure the semantic similarity of go terms using aggregate information content.', IEEE/ACM Trans Comput Biol Bioinform 11(3), 468-476.

Wu, X., Pang, E., Lin, K. \& Pei, Z.-M. (2013), 'Improving the measurement of semantic similarity between gene ontology terms and gene products: insights from an edgeand ic-based hybrid method.', PLoS One 8(5), e66745.

Wu, X., Zhu, L., Guo, J., Zhang, D.-Y. \& Lin, K. (2006), 'Prediction of yeast proteinprotein interaction network: insights from the gene ontology and annotations', $\mathrm{Nu}$ cleic acids research $\mathbf{3 4}(7)$, 2137-2150.

URL: https://www.ncbi.nlm.nih.gov/pubmed/16641319 


\section{Appendix}

\section{KEGG METABOLIC PATHWAY}

Table A.1: List of KEGG Metabolic Pathway

\begin{tabular}{lll}
\hline $\begin{array}{l}\text { KEGG } \\
\text { pathway }\end{array}$ & Pathway's name & $\begin{array}{l}\text { Number } \\
\text { of genes }\end{array}$ \\
\hline hsa00010 & Glycolysis / Gluconeogenesis & 68 \\
hsa00020 & Citrate cycle (TCA cycle) & 30 \\
hsa00030 & Pentose phosphate pathway & 30 \\
hsa00040 & Pentose and glucuronate interconversions & 34 \\
hsa00051 & Fructose and mannose metabolism & 33 \\
hsa00052 & Galactose metabolism & 31 \\
hsa00053 & Ascorbate and aldarate metabolism & 27 \\
hsa00061 & Fatty acid biosynthesis & 13 \\
hsa00062 & Fatty acid elongation & 30 \\
hsa00071 & Fatty acid degradation & 44 \\
hsa00072 & Synthesis and degradation of ketone bodies & 10 \\
hsa00100 & Steroid biosynthesis & 19 \\
hsa00120 & Primary bile acid biosynthesis & 17 \\
hsa00130 & Ubiquinone and other terpenoid-quinone biosynthesis & 11 \\
hsa00140 & Steroid hormone biosynthesis & 59 \\
hsa00190 & Oxidative phosphorylation & 133 \\
hsa00220 & Arginine biosynthesis & 21 \\
hsa00230 & Purine metabolism & 174 \\
hsa00232 & Caffeine metabolism & 5 \\
hsa00240 & Pyrimidine metabolism & 101 \\
hsa00250 & Alanine, aspartate and glutamate metabolism & 35 \\
hsa00260 & Glycine, serine and threonine metabolism & 40 \\
hsa00270 & Cysteine and methionine metabolism & 45 \\
hsa00280 & Valine, leucine and isoleucine degradation & 48 \\
hsa00290 & Valine, leucine and isoleucine biosynthesis & 4 \\
hsa00310 & Lysine degradation & 59 \\
hsa00330 & Arginine and proline metabolism & 50 \\
hsa00340 & Histidine metabolism & 23
\end{tabular}


hsa00350 Tyrosine metabolism 36

$\begin{array}{ll}\text { hsa00360 Phenylalanine metabolism } & 17\end{array}$

hsa00380 Tryptophan metabolism 40

hsa00400 Phenylalanine, tyrosine and tryptophan biosynthesis 5

hsa00410 beta-Alanine metabolism 31

hsa00430 Taurine and hypotaurine metabolism 11

$\begin{array}{lll}\text { hsa00440 } & \text { Phosphonate and phosphinate metabolism } & 6\end{array}$

$\begin{array}{lll}\text { hsa00450 Selenocompound metabolism } & 17\end{array}$

hsa00471 D-Glutamine and D-glutamate metabolism 5

hsa00472 D-Arginine and D-ornithine metabolism 1

hsa00480 Glutathione metabolism $\quad 56$

hsa00500 Starch and sucrose metabolism 36

$\begin{array}{lll}\text { hsa00510 N-Glycan biosynthesis } & 49\end{array}$

$\begin{array}{lll}\text { hsa00511 Other glycan degradation } & 18\end{array}$

hsa00512 Mucin type O-glycan biosynthesis 31

hsa00514 Other types of O-glycan biosynthesis 22

hsa00515 Mannose type O-glycan biosynthesis 23

hsa00520 Amino sugar and nucleotide sugar metabolism 48

hsa00524 Neomycin, kanamycin and gentamicin biosynthesis 5

hsa00531 Glycosaminoglycan degradation 19

hsa00532 Glycosaminoglycan biosynthesis - chondroitin sulfate / dermatan 20 sulfate

hsa00533 Glycosaminoglycan biosynthesis - keratan sulfate 14

hsa00534 Glycosaminoglycan biosynthesis - heparan sulfate / heparin 24

hsa00561 Glycerolipid metabolism $\quad 61$

hsa00562 Inositol phosphate metabolism $\quad 74$

hsa00563 Glycosylphosphatidylinositol (GPI)-anchor biosynthesis 25

hsa00564 Glycerophospholipid metabolism 97

$\begin{array}{lll}\text { hsa00565 Ether lipid metabolism } & 47\end{array}$

hsa00590 Arachidonic acid metabolism 63

hsa00591 Linoleic acid metabolism 29

hsa00592 alpha-Linolenic acid metabolism 25

hsa00600 Sphingolipid metabolism $\quad 47$

hsa00601 Glycosphingolipid biosynthesis - lacto and neolacto series $\quad 27$

hsa00603 Glycosphingolipid biosynthesis - globo and isoglobo series $\quad 15$

hsa00604 Glycosphingolipid biosynthesis - ganglio series $\quad 15$

hsa00620 Pyruvate metabolism 39

hsa00630 Glyoxylate and dicarboxylate metabolism 30

hsa00640 Propanoate metabolism $\quad 32$

hsa00650 Butanoate metabolism 28

hsa00670 One carbon pool by folate 20

$\begin{array}{lll}\text { hsa00730 Thiamine metabolism } & 16\end{array}$

hsa00740 Riboflavin metabolism 8 
hsa00750 Vitamin B6 metabolism 6

hsa00760 Nicotinate and nicotinamide metabolism 30

$\begin{array}{lll}\text { hsa00770 Pantothenate and CoA biosynthesis } & 19\end{array}$

hsa00780 Biotin metabolism 3

hsa00785 Lipoic acid metabolism 3

hsa00790 Folate biosynthesis $\quad 26$

$\begin{array}{lll}\text { hsa00830 Retinol metabolism } & 67\end{array}$

hsa00860 Porphyrin and chlorophyll metabolism 42

hsa00900 Terpenoid backbone biosynthesis 22

$\begin{array}{lll}\text { hsa00910 Nitrogen metabolism } & 17\end{array}$

hsa00920 Sulfur metabolism $\quad 9$

hsa00970 Aminoacyl-tRNA biosynthesis 66

hsa00980 Metabolism of xenobiotics by cytochrome P450 76

hsa00982 Drug metabolism - cytochrome P450 72

$\begin{array}{lll}\text { hsa00983 Drug metabolism - other enzymes } & 79\end{array}$

hsa01040 Biosynthesis of unsaturated fatty acids 23

hsa02010 ABC transporters 44

hsa03008 Ribosome biogenesis in eukaryotes 105

hsa03010 Ribosome 153

hsa03013 RNA transport 171

hsa03015 mRNA surveillance pathway $\quad 91$

$\begin{array}{lll}\text { hsa03018 RNA degradation } & 79\end{array}$

hsa03020 RNA polymerase 31

hsa03022 Basal transcription factors $\quad 45$

hsa03030 DNA replication 36

hsa03040 Spliceosome 134

hsa03050 Proteasome 45

hsa03060 Protein export $\quad 23$

hsa03320 PPAR signaling pathway 74

hsa03410 Base excision repair 33

hsa03420 Nucleotide excision repair $\quad 47$

hsa03430 Mismatch repair 23

hsa03440 Homologous recombination 41

hsa03450 Non-homologous end-joining 13

hsa03460 Fanconi anemia pathway $\quad 54$

hsa04010 MAPK signaling pathway 295

hsa04012 ErbB signaling pathway 85

hsa04014 Ras signaling pathway 232

hsa04015 Rap1 signaling pathway 206

hsa04020 Calcium signaling pathway 183

hsa04022 cGMP-PKG signaling pathway 163

hsa04024 cAMP signaling pathway 198

hsa04060 Cytokine-cytokine receptor interaction 294 
$\begin{array}{lll}\text { hsa04062 Chemokine signaling pathway } & 189\end{array}$

hsa04064 NF-kappa B signaling pathway $\quad 95$

hsa04066 HIF-1 signaling pathway 100

hsa04068 FoxO signaling pathway 132

$\begin{array}{lll}\text { hsa04070 } & \text { Phosphatidylinositol signaling system } & 99\end{array}$

$\begin{array}{lll}\text { hsa04071 Sphingolipid signaling pathway } & 118\end{array}$

hsa04072 Phospholipase D signaling pathway 146

$\begin{array}{ll}\text { hsa04080 Neuroactive ligand-receptor interaction } & 277\end{array}$

hsa04110 Cell cycle 124

hsa04114 Oocyte meiosis 124

hsa04115 p53 signaling pathway $\quad 72$

$\begin{array}{lll}\text { hsa04120 } & \text { Ubiquitin mediated proteolysis } & 137\end{array}$

hsa04122 Sulfur relay system 8

hsa04130 SNARE interactions in vesicular transport 34

hsa04136 Autophagy - other 32

hsa04137 Mitophagy - animal $\quad 65$

hsa04140 Autophagy - animal 128

hsa04141 Protein processing in endoplasmic reticulum 165

$\begin{array}{ll}\text { hsa04142 Lysosome } & 123\end{array}$

hsa04144 Endocytosis 244

hsa04145 Phagosome $\quad 152$

hsa04146 Peroxisome 83

hsa04150 mTOR signaling pathway 151

hsa04151 PI3K-Akt signaling pathway 354

$\begin{array}{ll}\text { hsa04152 AMPK signaling pathway } & 120\end{array}$

hsa04210 Apoptosis 136

hsa04211 Longevity regulating pathway 89

hsa04213 Longevity regulating pathway - multiple species 62

hsa04215 Apoptosis - multiple species 33

hsa04216 Ferroptosis $\quad 40$

hsa04217 Necroptosis 162

hsa04218 Cellular senescence 160

hsa04260 Cardiac muscle contraction 78

hsa04261 Adrenergic signaling in cardiomyocytes 144

hsa04270 Vascular smooth muscle contraction 121

$\begin{array}{ll}\text { hsa04310 Wnt signaling pathway } & 146\end{array}$

$\begin{array}{lll}\text { hsa04330 Notch signaling pathway } & 48\end{array}$

$\begin{array}{lll}\text { hsa04340 Hedgehog signaling pathway } & 47\end{array}$

hsa04350 TGF-beta signaling pathway 84

$\begin{array}{lll}\text { hsa04360 Axon guidance } & 175\end{array}$

$\begin{array}{lll}\text { hsa04370 } & \text { VEGF signaling pathway } & 59\end{array}$

$\begin{array}{ll}\text { hsa04371 Apelin signaling pathway } & 137\end{array}$

$\begin{array}{lll}\text { hsa04380 Osteoclast differentiation } & 128\end{array}$ 
hsa04390 Hippo signaling pathway 154

hsa04392 Hippo signaling pathway - multiple species 29

$\begin{array}{lll}\text { hsa04510 Focal adhesion } & 199\end{array}$

hsa04512 ECM-receptor interaction $\quad 82$

hsa04514 Cell adhesion molecules (CAMs) 144

$\begin{array}{lll}\text { hsa04520 Adherens junction } & 72\end{array}$

hsa04530 Tight junction 170

hsa04540 Gap junction $\quad 88$

hsa04550 Signaling pathways regulating pluripotency of stem cells $\quad 139$

hsa04610 Complement and coagulation cascades $\quad 79$

$\begin{array}{ll}\text { hsa04611 Platelet activation } & 123\end{array}$

$\begin{array}{lll}\text { hsa04612 Antigen processing and presentation } & 77\end{array}$

hsa04614 Renin-angiotensin system 23

hsa04620 Toll-like receptor signaling pathway 104

$\begin{array}{lll}\text { hsa04621 NOD-like receptor signaling pathway } & 168\end{array}$

hsa04622 RIG-I-like receptor signaling pathway 70

hsa04623 Cytosolic DNA-sensing pathway 63

hsa04625 C-type lectin receptor signaling pathway 104

hsa04630 JAK-STAT signaling pathway 162

hsa04640 Hematopoietic cell lineage $\quad 97$

hsa04650 Natural killer cell mediated cytotoxicity 131

hsa04657 IL-17 signaling pathway 93

hsa04658 Th1 and Th2 cell differentiation 92

$\begin{array}{lll}\text { hsa04659 Th17 cell differentiation } & 107\end{array}$

hsa04660 T cell receptor signaling pathway 101

hsa04662 B cell receptor signaling pathway 71

$\begin{array}{lll}\text { hsa04664 Fc epsilon RI signaling pathway } & 68\end{array}$

hsa04666 Fc gamma R-mediated phagocytosis $\quad 91$

hsa04668 TNF signaling pathway 110

hsa04670 Leukocyte transendothelial migration 112

hsa04672 Intestinal immune network for IgA production 49

hsa04710 Circadian rhythm 31

hsa04713 Circadian entrainment 96

$\begin{array}{ll}\text { hsa04714 Thermogenesis } & 229\end{array}$

$\begin{array}{ll}\text { hsa04720 Long-term potentiation } & 67\end{array}$

hsa04721 Synaptic vesicle cycle 63

$\begin{array}{ll}\text { hsa04722 Neurotrophin signaling pathway } & 119\end{array}$

hsa04723 Retrograde endocannabinoid signaling 148

hsa04724 Glutamatergic synapse 114

hsa04725 Cholinergic synapse 112

$\begin{array}{lll}\text { hsa04726 Serotonergic synapse } & 115\end{array}$

$\begin{array}{lll}\text { hsa04727 GABAergic synapse } & 88\end{array}$

hsa04728 Dopaminergic synapse 131 
$\begin{array}{lll}\text { hsa04730 } & \text { Long-term depression } & 60\end{array}$

$\begin{array}{ll}\text { hsa04740 Olfactory transduction } & 448\end{array}$

hsa04742 Taste transduction 83

hsa04744 Phototransduction 28

hsa04750 Inflammatory mediator regulation of TRP channels 99

hsa04810 Regulation of actin cytoskeleton 213

$\begin{array}{lll}\text { hsa04910 Insulin signaling pathway } & 137\end{array}$

hsa04911 Insulin secretion $\quad 85$

hsa04912 GnRH signaling pathway $\quad 93$

hsa04913 Ovarian steroidogenesis 49

hsa04914 Progesterone-mediated oocyte maturation 99

$\begin{array}{lll}\text { hsa04915 } & \text { Estrogen signaling pathway } & 137\end{array}$

hsa04916 Melanogenesis 101

$\begin{array}{lll}\text { hsa04917 Prolactin signaling pathway } & 70\end{array}$

$\begin{array}{lll}\text { hsa04918 Thyroid hormone synthesis } & 74\end{array}$

hsa04919 Thyroid hormone signaling pathway 116

hsa04920 Adipocytokine signaling pathway 69

hsa04921 Oxytocin signaling pathway 152

hsa04922 Glucagon signaling pathway 103

hsa04923 Regulation of lipolysis in adipocytes 54

$\begin{array}{lll}\text { hsa04924 Renin secretion } & 65\end{array}$

hsa04925 Aldosterone synthesis and secretion 96

$\begin{array}{lll}\text { hsa04926 Relaxin signaling pathway } & 130\end{array}$

$\begin{array}{lll}\text { hsa04927 Cortisol synthesis and secretion } & 64\end{array}$

hsa04928 Parathyroid hormone synthesis, secretion and action 106

hsa04930 Type II diabetes mellitus 46

$\begin{array}{lll}\text { hsa04931 Insulin resistance } & 107\end{array}$

hsa04932 Non-alcoholic fatty liver disease (NAFLD) 149

hsa04933 AGE-RAGE signaling pathway in diabetic complications 99

hsa04934 Cushing syndrome 154

hsa04940 Type I diabetes mellitus 43

hsa04950 Maturity onset diabetes of the young 26

hsa04960 Aldosterone-regulated sodium reabsorption 37

hsa04961 Endocrine and other factor-regulated calcium reabsorption $\quad 47$

hsa04962 Vasopressin-regulated water reabsorption 44

hsa04964 Proximal tubule bicarbonate reclamation 23

hsa04966 Collecting duct acid secretion $\quad 27$

$\begin{array}{ll}\text { hsa04970 Salivary secretion } & 90\end{array}$

$\begin{array}{lll}\text { hsa04971 Gastric acid secretion } & 75\end{array}$

hsa04972 Pancreatic secretion 96

hsa04973 Carbohydrate digestion and absorption 44

hsa04974 Protein digestion and absorption 90

hsa04975 Fat digestion and absorption 41 
hsa04976 Bile secretion $\quad 71$

hsa04977 Vitamin digestion and absorption 24

hsa04978 Mineral absorption $\quad 51$

hsa04979 Cholesterol metabolism 50

hsa05010 Alzheimer disease 171

hsa05012 Parkinson disease 142

hsa05014 Amyotrophic lateral sclerosis (ALS) 51

hsa05016 Huntington disease 193

hsa05020 Prion diseases $\quad 35$

hsa05030 Cocaine addiction 49

hsa05031 Amphetamine addiction 68

$\begin{array}{lll}\text { hsa05032 Morphine addiction } & 91\end{array}$

hsa05033 Nicotine addiction 40

hsa05034 Alcoholism 180

hsa05100 Bacterial invasion of epithelial cells 74

hsa05110 Vibrio cholerae infection 50

hsa05120 Epithelial cell signaling in Helicobacter pylori infection 68

hsa05130 Pathogenic Escherichia coli infection $\quad 55$

$\begin{array}{lll}\text { hsa05131 Shigellosis } & 65\end{array}$

hsa05132 Salmonella infection 86

$\begin{array}{lll}\text { hsa05133 Pertussis } & 76\end{array}$

hsa05134 Legionellosis $\quad 55$

$\begin{array}{lll}\text { hsa05140 Leishmaniasis } & 74\end{array}$

hsa05142 Chagas disease (American trypanosomiasis) 102

hsa05143 African trypanosomiasis 35

hsa05144 Malaria 49

$\begin{array}{ll}\text { hsa05145 Toxoplasmosis } & 113\end{array}$

$\begin{array}{lll}\text { hsa05146 Amoebiasis } & 96\end{array}$

hsa05150 Staphylococcus aureus infection $\quad 56$

hsa05152 Tuberculosis 179

hsa05160 Hepatitis C 131

hsa05161 Hepatitis B 144

hsa05162 Measles 132

hsa05163 Human cytomegalovirus infection 225

$\begin{array}{lll}\text { hsa05164 Influenza A } & 171\end{array}$

hsa05165 Human papillomavirus infection 339

hsa05166 Human T-cell leukemia virus 1 infection 255

hsa05167 Kaposi sarcoma-associated herpesvirus infection 186

hsa05168 Herpes simplex infection 185

hsa05169 Epstein-Barr virus infection 200

hsa05170 Human immunodeficiency virus 1 infection 212

hsa05202 Transcriptional misregulation in cancer 186

hsa05203 Viral carcinogenesis 201 
hsa05204 Chemical carcinogenesis $\quad 82$

hsa05205 Proteoglycans in cancer 201

hsa05206 MicroRNAs in cancer 299

hsa05210 Colorectal cancer 86

hsa05211 Renal cell carcinoma $\quad 69$

hsa05212 Pancreatic cancer $\quad 75$

hsa05213 Endometrial cancer 58

hsa05214 Glioma $\quad 71$

$\begin{array}{lll}\text { hsa05215 } & \text { Prostate cancer } & 97\end{array}$

hsa05216 Thyroid cancer $\quad 37$

hsa05217 Basal cell carcinoma 63

$\begin{array}{lll}\text { hsa05218 Melanoma } & 72\end{array}$

hsa05219 Bladder cancer 41

hsa05220 Chronic myeloid leukemia 76

hsa05221 Acute myeloid leukemia 66

hsa05222 Small cell lung cancer $\quad 93$

hsa05223 Non-small cell lung cancer 66

hsa05224 Breast cancer 147

hsa05225 Hepatocellular carcinoma 168

hsa05226 Gastric cancer 149

hsa05230 Central carbon metabolism in cancer 65

hsa05231 Choline metabolism in cancer 99

hsa05310 Asthma 31

hsa05320 Autoimmune thyroid disease 53

hsa05321 Inflammatory bowel disease (IBD) 65

hsa05322 Systemic lupus erythematosus 133

$\begin{array}{lll}\text { hsa05323 Rheumatoid arthritis } & 90\end{array}$

hsa05330 Allograft rejection 38

hsa05332 Graft-versus-host disease 41

hsa05340 Primary immunodeficiency 37

hsa05410 Hypertrophic cardiomyopathy (HCM) 83

hsa05412 Arrhythmogenic right ventricular cardiomyopathy (ARVC) 72

hsa05414 Dilated cardiomyopathy (DCM) 90

$\begin{array}{lll}\text { hsa05416 Viral myocarditis } & 59\end{array}$

$\begin{array}{lll}\text { hsa05418 Fluid shear stress and atherosclerosis } & 139\end{array}$ 